\title{
LA PUBLICIDAD, UNA ESTRATEGIA DE ÉXITO PARA LAS MICRO, PEQUEÑAS Y MEDIANAS EMPRESAS DE LA REGIÓN CENTRO Y SUROESTE DEL ESTADO DE HIDALGO ${ }^{1}$
}

\author{
THE ADVERTISING, A STRATEGY OF SUCCESS FOR MICRO, SMALL AND \\ MEDIUM ENTERPRISES IN THE CENTRAL AND SOUTHWEST REGION IN \\ STATE HIDALGO
}

\section{Ruth Flores Jiménez ${ }^{2}$ \\ Ivette Flores Jiménez ${ }^{3}$, Raúl Rodríguez Moreno ${ }^{4}$ Miguel Ángel Vázquez Alamilla 5}

Para citar este artículo: Flores, R., Flores, I., Rodríguez, R. y Vásquez, M. (2014). “La publicidad, una estrategia de éxito para las micro, pequeñas y medianas empresas de la región centro y suroeste del Estado de Hidalgo". Inquietud Empresarial. Vol. XIV (2), p.p. 133-152

Fecha de recepción: 2 de agosto de 2014 Fecha de Aceptación: 17 de noviembre de 2014

\footnotetext{
1 Artículo de reflexión como resultado del proyecto de investigación titulado "Diagnóstico de requerimientos de actualización de las funciones administrativas ( Personal, comerciales y de marketing, contables y financieras, seguridad, calidad, producción y productividad, informática administrativa) en las pequeñas y medianas empresas en la región suroeste y centro del estado de Hidalgo".

2 Maestra en Administración. Universidad Autónoma del estado de Hidalgo. Escuela Superior Tlahuelilpan. Grupo de investigación: Administración estratégica y Toma de decisiones. País. México. ruthfj_22@hotmail.com

3 Maestra en Administración de Negocios. Universidad Autónoma del estado de Hidalgo. Escuela Superior Tlahuelilpan. Grupo de investigación: Administración estratégica y Toma de decisiones. ivette@uaeh.edu.mx ivette_flores7@hotmail.com

4 Universidad Autónoma del estado de Hidalgo. Escuela Superior Tlahuelilpan. Grupo de investigación: Administración estratégica y Toma de decisiones. raromo@hotmail.com.

5 Universidad Autónoma del estado de Hidalgo. Escuela Superior Tlahuelilpan. Grupo de investigación: Administración estratégica y Toma de decisiones. m_vazquez_alamilla@hotmail.com.
} 


\section{RESUMEN}

Las empresas que buscan superarse deben prepararse para desarrollar programas integrales que exijan formas inteligentes y responsables de competitividad y productividad como parte del éxito en mercados globalizados. El crecimiento de grandes empresas mexicanas se basa en la preparación y actualización profesional de su personal y expansión del conocimiento.

El objetivo del presente trabajo: 1) identificar los principales desafíos en capacitación de MiPyMES y las demandas hacia las instituciones de educación superior y 2) formular recomendaciones de comercialización orientadas a mejorar procesos de mercadotecnia.

\section{PALABRAS CLAVE}

MyPYMES, marketing publicitario.

\section{ABSTRACT}

Companies must be prepared to develop comprehensive programs that require intelligent and responsible forms of competitiveness and productivity to be a part of success companies in global markets. The growth of large Mexican companies is based on the professional development of its staff and the expansion of knowledge.

The aims of this study are: 1) identify the training key challenges for MSMEs and the training demands to higher education institutions and 2) propose marketing recommendations aimed to improve the marketing processes.

\section{KEYWORDS}

MSMEs, advertising marketing.

\section{INTRODUCCIÓN}

Considerando estos aspectos podemos identificar las deficiencias en gran parte de las MiPyMEs de esta región basándonos en su respuesta, de igual manera que podemos observar el funcionamiento de estas, apoyándonos en el análisis de los datos arrojados, donde la mayoría de las MiPyMEs pertenecen al sector comercial y cuentan con un número de trabajadores entre uno y diez, de esta manera nos podemos percatar de la magnitud del problema, ya que estas MiPyMEs son muy pequeñas, por lo tanto deben tener unas ventas regulares o muy bajas que solo les permiten sostenerse (porque de otra manera tendrían una mayor expansión y un mayor número de empleados), un problema de ello puede ser a la falta de administración, de manera que solicitan capacitación en modelos de gestión administrativa

Esta situación obliga a las empresas a un replanteo organizativo y económico que va más allá de un ajuste en su trayectoria evolutiva. Es decir, la transformación requerida por las nuevas circunstancias no se corresponde mecánicamente con una fase posterior de desarrollo alcanzado por las empresas. Por lo tanto, en el nuevo escenario de globalización, apertura y reformas estructurales, el desarrollo de capacidades innovativas, donde la capacitación y actualización profesional ocupan 
LA PUBLICIDAD, UNA ESTRATEGIA DE ÉXITO PARA LAS MICRO, PEQUEÑAS Y MEDIANAS EMPRESAS DE LA REGIÓN CENTRO Y SUROESTE DEL ESTADO DE HIDALGO

un espacio importante, se convierte en un punto clave para la búsqueda de una mayor competitividad.

En ese contexto, el objetivo de este trabajo es: 1) identificar los principales desafíos que en materia de capacitación enfrentan las Pequeñas y medianas empresas (Pymes) y las demandas hacia las instituciones del sistema científico y tecnológico; o sea las Instituciones de Educación superior, 2) analizar el grado de vinculación de las instituciones a las demandas de las firmas y 3) formular estrategias de mercadeo orientadas a mejorar la comercialización entre ellas. Para lograr una atención más eficiente de los requerimientos que en materia de crecimiento requieren.

Para detectar los desafíos y demandas que en materia de capacitación enfrentan las (MiPymes) se realizará una investigación que se iniciará en la zona del Valle del Mezquital y Centro del estado de Hidalgo con entrevistas y visitas a empresas de este tamaño. La metodología utilizada está orientada a recabar la información relacionada con sus necesidades de capacitación y actualización profesional con el objetivo de efectuar un análisis ordenado de los participantes (Pymes) y realizar un informe documentado y grupal que permita la definición de programas de capacitación de acuerdo con las necesidades planteadas. Esto permitirá en general, el impulso elevado del posicionamiento competitivo y representativo del espacio productivo MiPyme que sean o hayan sido en los últimos años demandantes de Capacitación y actualización profesional para sus trabajadores, lo que les permitirá un importante desarrollo

\section{Las MIPYMES en la economía nacional en México}

En México la mayoría de las empresas son Micro, Pequeñas y Medianas (como se puede observar en la Figura 1.) según estudios previos realizados por la SE.

Figura 1. Estructura de las PYMES en México.

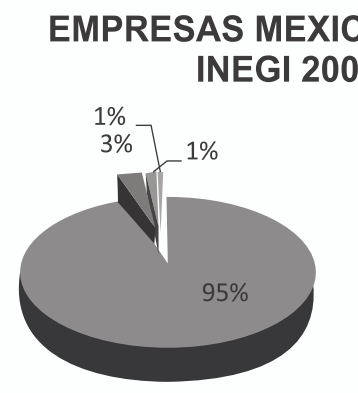

\section{CENSO}

\section{9}

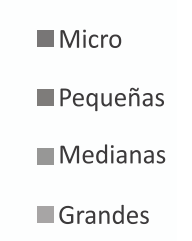

\section{PERSONAS OCUPADAS POR CLASIFICACIÓN DEL TIPO DE EMPRESA CENSO MEXICO 2009}

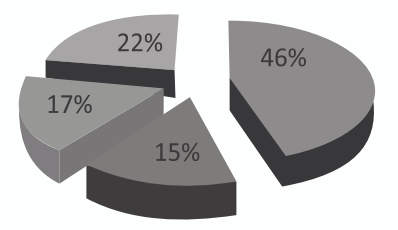

Micro

- Pequeñas

Medianas

Grandes

Fuente: Secretaría de Economía (2012)

De acuerdo con el Instituto nacional de estadística y geografía (2009), el número de establecimientos empresariales en México arroja que existen 5'144,056 unidades económicas en el país, (Ver cuadro 4.); los cuales generan 27, 727,406 millones de empleos (Ver cuadro 5). De éstos las MIPYMES representaron el 78\% del empleo y el 50 \% del PIB, destacando la necesidad de una mayor atención a este sector de micros y medianos empresarios. 
Tabla 1. Unidades económicas existentes en 2009, por año de inicio de actividades según actividad económica

\begin{tabular}{|c|c|c|c|c|c|c|}
\hline \multirow{2}{*}{$\begin{array}{l}\text { Actividad } \\
\text { Económica }\end{array}$} & \multicolumn{2}{|l|}{ Total } & \multicolumn{2}{|c|}{$\begin{array}{l}\text { Realizaron actividades } \\
\text { en } 2008\end{array}$} & \multicolumn{2}{|c|}{$\begin{array}{l}\text { Iniciaron actividades en } \\
2009\end{array}$} \\
\hline & Absoluto & $\%$ & Absoluto & $\%$ & Absoluto & $\%$ \\
\hline Total & $5,144,056$ & 100.0 & $4,724,892$ & 100.0 & 419,164 & 100.0 \\
\hline Comercio & $2,424,249$ & $47.1 \%$ & $2,205,239$ & 46.7 & 219,010 & 52.2 \\
\hline $\begin{array}{l}\text { Servicios } \\
\text { privados no } \\
\text { financieros }\end{array}$ & $2,056,437$ & 40.0 & $1,894,244$ & 40.1 & 162,193 & 38.7 \\
\hline $\begin{array}{l}\text { Industrias } \\
\text { manufactureras }\end{array}$ & 581044 & 11.3 & 545372 & 11.5 & 35672 & 8.5 \\
\hline $\begin{array}{l}\text { Servicios } \\
\text { financieros y de } \\
\text { seguro }\end{array}$ & 20049 & 0.4 & 18706 & 0.4 & 1343 & 0.3 \\
\hline $\begin{array}{l}\text { Pesca y } \\
\text { acuicultura }\end{array}$ & 19454 & 0.4 & 19443 & 0.4 & 11 & 0.0 \\
\hline Construcción & 19020 & 0.4 & 18637 & 0.4 & 383 & 0.1 \\
\hline $\begin{array}{l}\text { Transportes, } \\
\text { correos y } \\
\text { almacenamiento }\end{array}$ & 18257 & 0.4 & 17705 & 0.4 & 552 & 0.1 \\
\hline Minería & 2957 & 0.1 & 2957 & 0.1 & 0 & 0.0 \\
\hline $\begin{array}{l}\text { Electricidad, } \\
\text { agua y gas }\end{array}$ & 2589 & 0.1 & 2589 & 0.1 & 0 & 0.0 \\
\hline
\end{tabular}

Fuente: Instituto nacional de estadística y geografía (2009)

Tabla 2. Personal Ocupado por las Unidades Económicas existentes en 2009

\begin{tabular}{|l|c|c|}
\hline & \multicolumn{1}{|c|}{$\begin{array}{c}\text { Unidades } \\
\text { económicas }\end{array}$} & Personal ocupado \\
\hline $\begin{array}{l}\text { Universo de unidades } \\
\text { económicas }\end{array}$ & 5144056 & 27727406 \\
\hline $\begin{array}{l}\text { Realizaron actividades en el } \\
2008\end{array}$ & 4724892 & 26863014 \\
\hline Iniciaron actividades en el 2008 & 419164 & 864392 \\
\hline
\end{tabular}

Fuente: Sistema de Información Empresarial Mexicana (2010)

Las fuentes con mayor confiabilidad, de acuerdo con sus metodologías de contabilización son: el Instituto Mexicano del Seguro Social (IMSS), el Sistema 
LA PUBLICIDAD, UNA ESTRATEGIA DE ÉXITO PARA LAS MICRO, PEQUEÑAS Y MEDIANAS EMPRESAS DE LA REGIÓN CENTRO Y SUROESTE DEL ESTADO DE HIDALGO

Empresarial Mexicano (SIEM) de la Secretaría de Economía (SE) y el Instituto Nacional de Estadística Geografía (INEGI).

El IMSS utiliza el concepto de "patrones", el cual incluye a personas físicas y morales que tienen registrado al menos a un trabajador asalariado. Dentro de estos patrones pueden existir personas que no tienen actividades empresariales, como amas de casa. En el caso del INEGI, existen duplicidades, no cuantificadas, en el número de empresas, dado que algunas compañías cuentan con más de un establecimiento.

Para objeto de este estudio, no todas las empresas pueden ser catalogadas como capacitables, nos referimos en lo particular a las microempresas ya que... "muchas de ellas se constituyen en una actividad de supervivencia, probablemente resultado de las diversas crisis económicas recurrentes e incluso de la incapacidad del sector formal de absorber la creciente oferta de trabajo, o que obliga a quienes no pueden ingresar al mercado laboral a generar su propio ingreso" (Nacional financiera, 2001).

Una parte, no cuantificada, se concentra en actividades como centros nocturnos y bares que no serían sujetas de financiamiento para capacitación por parte de agencias gubernamentales o universidades.

Por lo anterior éste trabajo se concentró en lo fundamental, en las PYMES del sector industrial.

\section{Algunos problemas de la capacitación de las PYMES}

La capacitación en México enfrenta, entre otros, los problemas siguientes:

"Enfoque de la capacitación dominado por la oferta. Esto significa, que los programas y cursos se diseñan, aplican y evalúan desde la academia, los centros encargados de la capacitación, áreas de recursos humanos de las empresas, con lo cual no necesariamente se están atendiendo los requerimientos de los procesos productivos modernos " (Ibarra, 1999).

"Fragmentación institucional y escasa coordinación entre programas. Cuando se detecta una nueva demanda, a veces hasta tardíamente, se busca atenderla, a través de la creación de una institución.... se atomizan los cursos, la oferta de capacitación es fragmentada, sin vinculación entre las instituciones que desarrollan la capacitación" (Ibarra, 1999).

"La incompatibilidad entre la organización de los servicios de capacitación por especialidades y las actuales demandas de la planta productiva. En el mundo del trabajo es cada vez más frecuente el cambio en los perfiles ocupacionales....analizar y resolver problemas, trabajar en equipo. De ahí que programas de capacitación organizados por especialidad no permitan atender con calidad y pertinencia esas necesidades de formación" (Ibarra, 1999).

Rigidez de programas. En el sistema tradicional, la formación se desarrolla mediante programas extensos que carecen de flexibilidad, entre otras razones, porque fueron diseñados con una sola entrada (inicio) y una sola salida (fin). 


\section{METODOLOGÍA}

\section{Planteamiento del problema}

La inexistencia o implementación de programas de capacitación deficientes, provoca bajos niveles de competitividad a las PyMEs de la región suroeste y centro del estado de Hidalgo.

\section{Justificación}

Siendo la capacitación un factor importante en el campo empresarial, entendida esta como un proceso de formación social, a través del cual los hombres se informan en el medio en que vive, a la vez que se capacitan para aplicar dicha información en su realidad circundante con el objeto de influir en ella; es interesante resaltar que las organizaciones siempre deberán tener un objetivo, ya que no se educa para un mundo abstracto, sino para participar de una manera u otra dentro de una actividad de la cual se forma parte. Es aquí donde se liga el aspecto educativo con el laboral, si se considera que el hombre es plenamente social cuando pasa formalmente de la fuerza productiva de un país, para lo cual habrá de utilizar los conocimientos adquiridos durante su proceso de capacitación.

En este sentido, habrá de considerarse que no toda información que se adquiere dentro del proceso de capacitación formal proporciona los medios necesarios para adaptarse íntegramente a las condiciones de trabajo; esto es, no todos los conocimientos adquiridos durante la fase de permanencia en la empresa, facilitan a los individuos su acceso a un puesto de trabajo, por lo que se hace necesario, establecer un puente entre el aspecto educativo y el aparato productivo. Esto exige un perfeccionamiento constante en la medida en que se desarrolla la tecnología y evolucionan las formas de producción, por lo que se puede concluir que la capacitación regular proporciona los conocimientos generales a la vez que desarrolla la capacidad mental básica, mientras que la capacitación en el trabajo, tiene como función primordial el desarrollo de especializaciones cambiantes y redefinidas de acuerdo con las necesidades del desarrollo social y económico.

En el estado de Hidalgo el adiestramiento es un elemento necesario, de acuerdo con los resultados de otras investigaciones, como por ejemplo las señaladas por Martínez (2008) en donde se refleja la preocupación por la capacitación por parte de los empresarios hidalguenses es incipiente, ya que no se conocen programas específicos de formación para el trabajo y lograr que las empresas del estado de Hidalgo tengan un mayor desarrollo, ya que a través de la capacitación crecería la productividad empresarial, sin embargo, la dimensión de las unidades económicas del estado de Hidalgo conformadas por micro y pequeñas empresas ocupan la mayor parte, con salarios mínimos, y nulas prestaciones, y no permiten tener un presupuesto adicional para el rubro de capacitación, eliminando oportunidades de crecimiento y desarrollo empresarial. Por otro lado se presentan oportunidades de regularización de negocios, ordenamientos, reorganizaciones, reestructuras, y todo tipo de aspectos relacionados con el fortalecimientos de estas actividades económicas, mediante la capacitación de personal para su conservación, defensa y competitividad y el actual entorno de supervivencia por lo que este tipo de organizaciones se ve afectado, así como el resurgimiento del conjunto de empresas y microempresas de la región, sin que se logre atraer la inversión extranjera, que permita despegar nuevamente a la región. Lo más grave es que se pierde una generación de mano de obra experta en procesos industriales, por lo que es necesario diagnosticar el proceso de capacitación de acuerdo con su área de desarrollo para la solución a una 
LA PUBLICIDAD, UNA ESTRATEGIA DE ÉXITO PARA LAS MICRO, PEQUEÑAS Y MEDIANAS EMPRESAS DE LA REGIÓN CENTRO Y SUROESTE DEL ESTADO DE HIDALGO

problemática que hace que las empresas no puedan ser competitivas.

Estos aspectos se reflejan en los municipios del estado de Hidalgo seleccionados para la presente investigación, que de acuerdo con el desarrollo geográfico del estado son los que tienen mayor participación económica por su aportación al PIB estatal, en ellos se reflejan, los diversos sectores económicos; industrial, comercial y de servicios que conforman la entidad y que han sido seleccionados para efecto de este proyecto de investigación

De lo expuesto anteriormente podemos concluir la importancia de la capacitación y que se concibe como el conjunto de acciones tendientes a proporcionar y/o a desarrollar actitudes de una persona, con el fin de prepararlo para que desempeñe adecuadamente su ocupación o puesto de trabajo y /o los inmediatos superiores de cualquier organización, permitiendo la implantación de nuevos programas de capacitación acordes a las necesidades de cada una de las micro y pequeñas empresas de las zonas anteriormente señaladas.

\section{Objetivos}

\section{- General}

Identificar y definir cuál es la necesidad de capacitación en las PyMEs de la región suroeste y centro del estado de Hidalgo, considerando los aspectos geográficos, demográficos, económicos, sociales, políticos y culturales en donde se encuentran, a fin de conocer la problemática existente en éste tópico, y proponer alternativas de comercialización publicitaria que les permitan lograr niveles superiores de competitividad.

\section{- Específicos}

1. Distinguir los diferentes tipos y áreas de capacitación que se ofrecen en las PyMEs de la región suroeste del estado de Hidalgo.

2. Señalar los niveles y la frecuencia en que se ofrece la capacitación en las PyMEs de la región suroeste del estado de Hidalgo.

3. Determinar cuáles son los instrumentos implementados para evaluar el impacto de la capacitación que se ofrecen en las PyMEs de la región suroeste del estado de Hidalgo.

4. Proponer herramientas de comercialización publicitarias como estrategia de crecimiento para las micro, pequeñas y medianas empresas de la región centro y suroeste del estado de Hidalgo.

\section{Selección de la muestra}

Se cuenta con un catálogo de empresas con un universo de 234 , las cuales son objeto de la investigación.

\section{Determinación del método de muestreo}

Se seleccionaron del formato de las 234 empresas más importantes de la región, más dicha muestra debería reunir ciertas características que nos permitieran alcanzar nuestro objetivo. Tendría que ser representativa del universo, a su vez suficiente en 
elementos seleccionados. En cuanto a las ventajas de utilizar muestras y no todo el universo es el tiempo que se invierte, la reducción del costo de la investigación y la confiabilidad al ser comprobada la representatividad de la muestra.

El cálculo de la muestra se realiza a través de dos fórmulas una para poblaciones infinitas (más de 500000 elementos) y otra para poblaciones finitas (menos de 500 000 elementos).

En nuestro caso se utilizó la fórmula para población finita, ya que nuestro universo sólo consta de 234 elementos.

\section{Determinación de la muestra}

Una vez que tenemos nuestro universo de 234 empresas de la región podemos determinar la muestra.

Para poblaciones finitas se utiliza la siguiente fórmula:

$$
\mathrm{n}=\frac{\mathrm{z}^{2} \mathrm{nP} \mathrm{Q}}{\mathrm{e}^{2}(\mathrm{n}-1)+\mathrm{z}^{2} \mathrm{p} \mathrm{q}}
$$

En donde:

$z=$ nivel de confianza

$\mathrm{N}=$ universo (anexo no. 3)

$\mathrm{p}=$ probabilidad a favor

$\mathrm{q}=$ probabilidad en contra

$\mathrm{e}=$ error de estimación (precisión en los resultados)

$\mathrm{n}=$ número de elementos (tamaño de la muestra)

Datos:

$z=95 \%, 1.96$

$\mathrm{N}=234$

$\mathrm{p}=50 \%$

$q=50 \%$

$\mathrm{e}=5 \%$

Tamaño de la muestra: 146 empresas. 
LA PUBLICIDAD, UNA ESTRATEGIA DE ÉXITO PARA LAS MICRO, PEQUEÑAS Y MEDIANAS EMPRESAS DE LA REGIÓN CENTRO Y SUROESTE DEL ESTADO DE HIDALGO

\section{RESULTADOS}

Figura 2. Sectores a los que pertenecen las Pymes

\section{SECTORES A LOS QUE PERTENECEN LAS PYMES}

$\begin{array}{ll}\text { Sector Comercial } & \begin{array}{l}\text { De los sectores a los que } \\ \text { pertenecen las PYMES el } \\ 57 \% \text { pertenece al sector } \\ \text { comercial, } 28 \% \text { al sector } \\ \text { Sector Servicios }\end{array} \\ \begin{array}{l}\text { de servicios, en un } 8 \% \text { al } \\ \text { ind u st r i a I, 2\% a I }\end{array} \\ \text { Sector Industrial } & \begin{array}{l}\text { agropecuario, 3\% a otro } \\ \text { tipo diferente y un 2\% no }\end{array} \\ \text { Sector Agropecuario } & \text { mencionaron sector }\end{array}$

No mencionaron sector

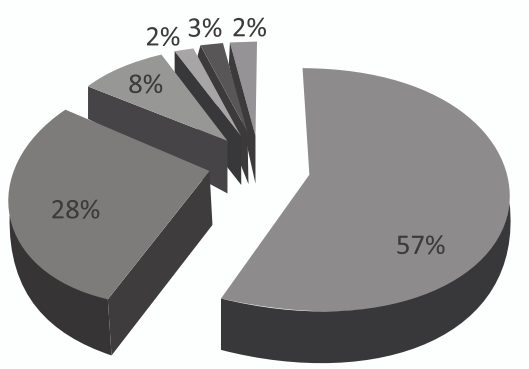

Fuente: Elaboración propia

Más de la mitad de las empresas encuestadas pertenecen al sector comercial, seguida del sector servicios y solo en un porcentaje menor pertenece al sector servicios.

Figura 3. Número de trabajadores

NUM. DE TRABAJADORES

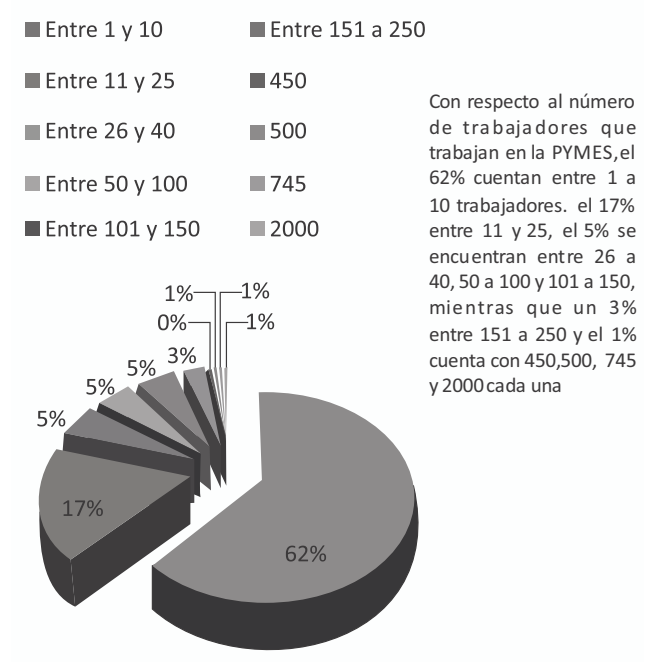

Fuente: Elaboración propia 
Se observa que en un porcentaje alto las Pymes analizadas tienen menos de 10 trabajadores, solamente el 1\% de las empresas tienen más de 450 trabajadores, lo que ratifica que en su mayoría son microempresas.

Figura 4. Servicios requeridos de capacitación en el ámbito de la Mercadotecnia y la Administración.

\section{SERVICIO REQUERIDOS DE CAPACITACION EN EL AMBITO DE LA MERCADOTECNICA Y LA ADMINISTRACION}

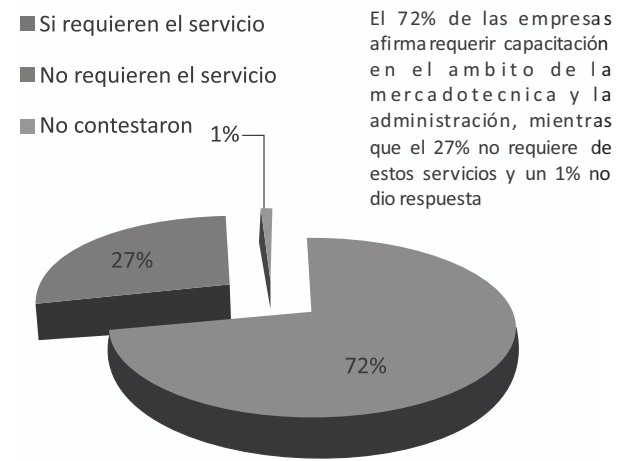

Fuente: Elaboración propia

Para las empresas encuestadas, en su mayoría la capacitación debe orientarse en el área de la mercadotecnia y la administración, el restante de las empresas afirmo no requerir capacitación de ninguna índole.

Figura 5. Empresas que utilizan los servicios de capacitación en el ámbito de la Mercadotecnia y la Administración

\section{EMPRESAS DE UTILIZADO SERVICIOS DE CAPACITACIÓN EN EL ÁMBITO DE LA MERCADOTECNIA Y LA ADMINISTRACIÓN}

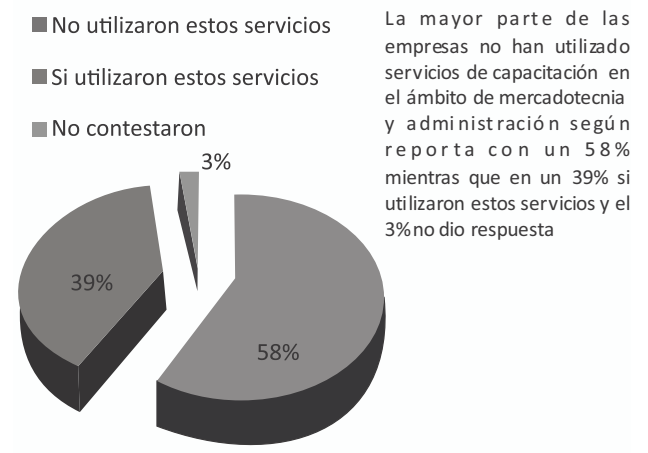

Fuente: Elaboración propia 
LA PUBLICIDAD, UNA ESTRATEGIA DE ÉXITO PARA LAS MICRO, PEQUEÑAS Y MEDIANAS EMPRESAS DE LA REGIÓN CENTRO Y SUROESTE DEL ESTADO DE HIDALGO

La mayor parte de las empresas no han utilizado servicios de capacitación en el ámbito de mercadotecnia y administración (58\%), el restante afirmo utilizar estos servicios.

Figura 6. Instrumentos empleados por las Pymes para evaluar el impacto de la capacitación

\section{INSTRUMENTOS EMPLEADOS POR LAS \\ PYMES PARA EVALUAR EL IMPACTO DE LA CAPACITACION}

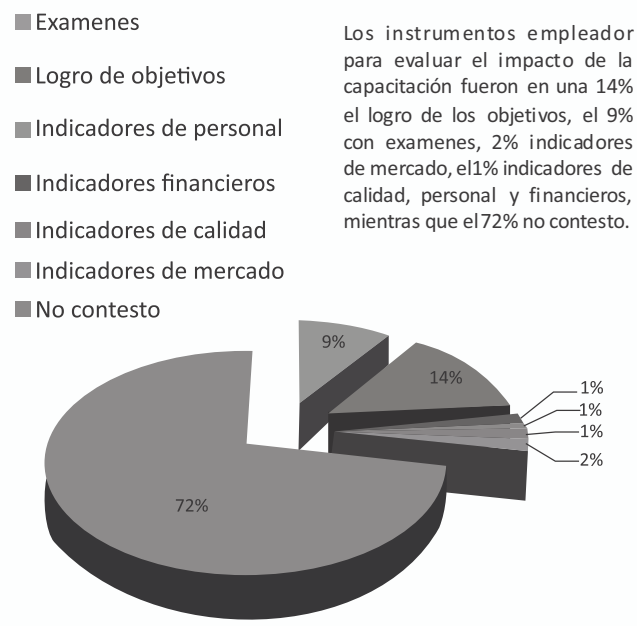

Fuente: Elaboración propia

Figura 7. Disponibilidad de las empresas para incorporar un programa de capacitación y desarrollo en materia de administración y mercadotecnia

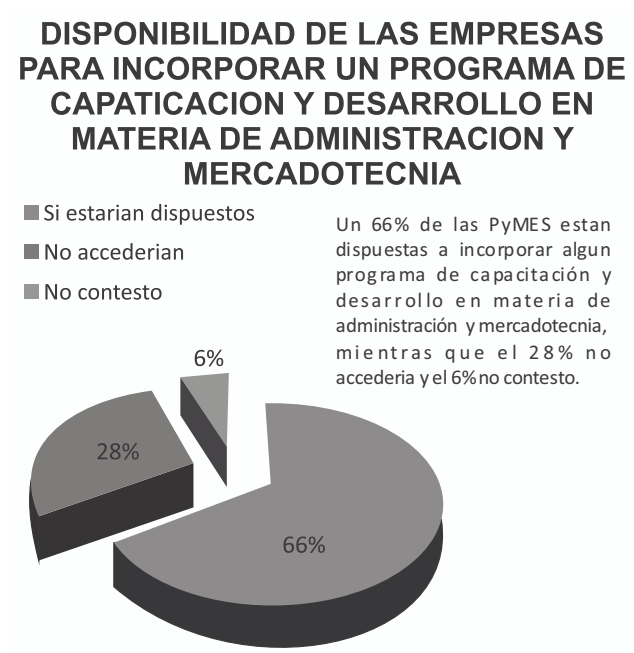

Fuente: Elaboración propia 
Figura 8. Personal que mayor capacitación recibe según área

\title{
PERSONAL QUE MAYOR CAPACITACIÓN RECIBE SEGÚN EL ÁREA
}

\author{
Personal \\ - Finanzas \\ - Mercadotecamiia \\ - Produccion \\ Calidad \\ - Otras Án \\ No contestaron
}

El personal que mayor capacitación recibe en el área de personal es de $13 \%$, en finanzas y mercadotecnia un $5 \%$, en producción y otras áreasun $3 \%$ y en un $1 \%$ en calidad

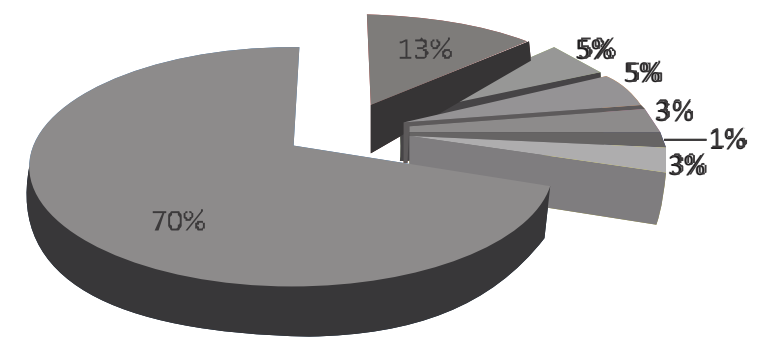

Fuente: Elaboración propia

Figura 9. Importancia que dan las Pymes a las necesidades de capacitación en materia legal

\section{IMPORTANCIA QUE DAN LAS PYMES A LAS NECESIDADES DE CAPATICACION EN MATERIA LEGAL}

$\begin{array}{ll}\text { Derecho Laboral } & \begin{array}{l}\text { El mayor grado de importancia que } \\ \text { las PYMES le da a la capacitacion en } \\ \text { materialegal es paraderecho laboral }\end{array} \\ \begin{array}{l}\text { Derecho Mercamtill } \\ \text { Derecho Constitucionall }\end{array} & \begin{array}{l}\text { con un } 32.5 \%, \text { un } 25.5 \% \text { derecho } \\ \text { mercantil, } 12.5 \% \text { para derech o }\end{array} \\ \text { y Administrativo } & \text { constitucional y administrativo. } \\ \text { Derecho Fiscal } & 14.5 \% \text { para de recho fiscal, } 0.5 \% \\ \text { otros } & \text { otros mientras que el } 15 \% \text { no } \\ \text { contesto. }\end{array}$

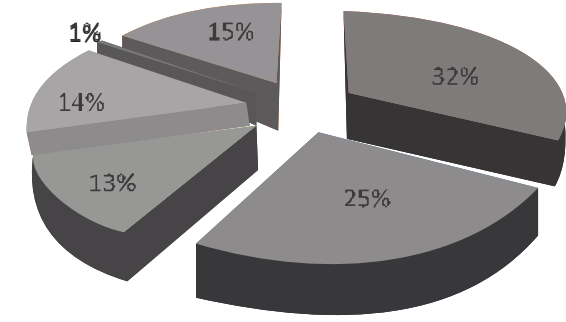

Fuente: Elaboración propia 
LA PUBLICIDAD, UNA ESTRATEGIA DE ÉXITO PARA LAS MICRO, PEQUEÑAS Y MEDIANAS EMPRESAS DE LA REGIÓN CENTRO Y SUROESTE DEL ESTADO DE HIDALGO

Figura 10. Importancia que dan las Pymes a las necesidades de capacitación en materia de administración general

\section{IMPORTANCIA QUE DAN LAS PYMES \\ A LAS NECESIDADES DE CAPACITACION \\ EN MATERIA DE ADMON. GENERAL}

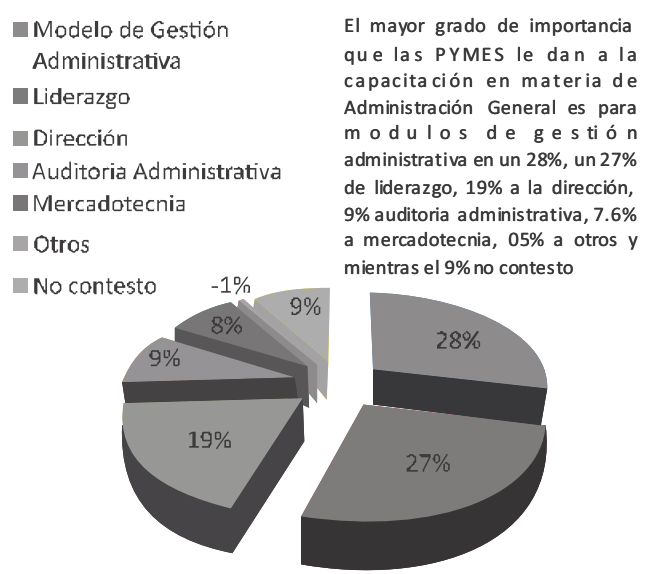

Fuente: Elaboración propia

Figura 11. Importancia que dan las Pymes a las necesidades de capacitación en materia contable

\section{IMPORTANCIA QUE DAN LA PYMES \\ A LAS NECESIDADES DE CAPACITACION EN MATERIA CONTABLE}

$\begin{array}{ll}\text { Contabilidad Básica } & \text { El mayor grado de importancia } \\ \text { Control Interno } & \begin{array}{l}\text { que las PYMES les dan a la } \\ \text { capacitación en materia contable } \\ \text { es a contabilidad básica con un }\end{array} \\ \text { Costos } & 33.2 \% \text {, seguida de control interno } \\ \text { Presupuestos } & \text { con un } 14.10 \%, \text { un } 11.10 \text { a costos, } \\ \text { - Finanzas } & \text { un } 8.5 \% \text { presupuestos, un } 8 \% \text { a } \\ \text { Economía } & \text { finanzas, un } 6 \% \text { a econimia, un } 4 \% \\ \square \text { impuestos } & \text { contesto. } \\ \text { No contesto } & \end{array}$
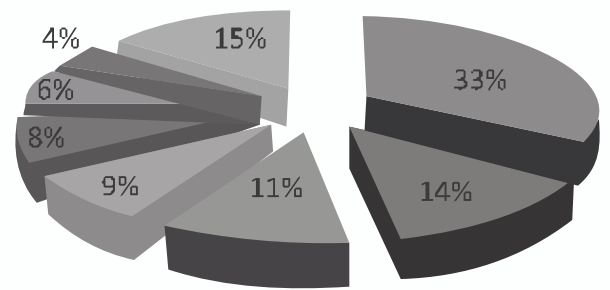

Fuente: Elaboración propia 
Figura 12. Importancia que dan las Pymes a las necesidades de capacitación en materia de personal

\section{IMPORTANCIA QUE DAN LAS PYMES A LAS NECESIDADES DE CAPACITACION EN MATERIA DE PERSONAL}

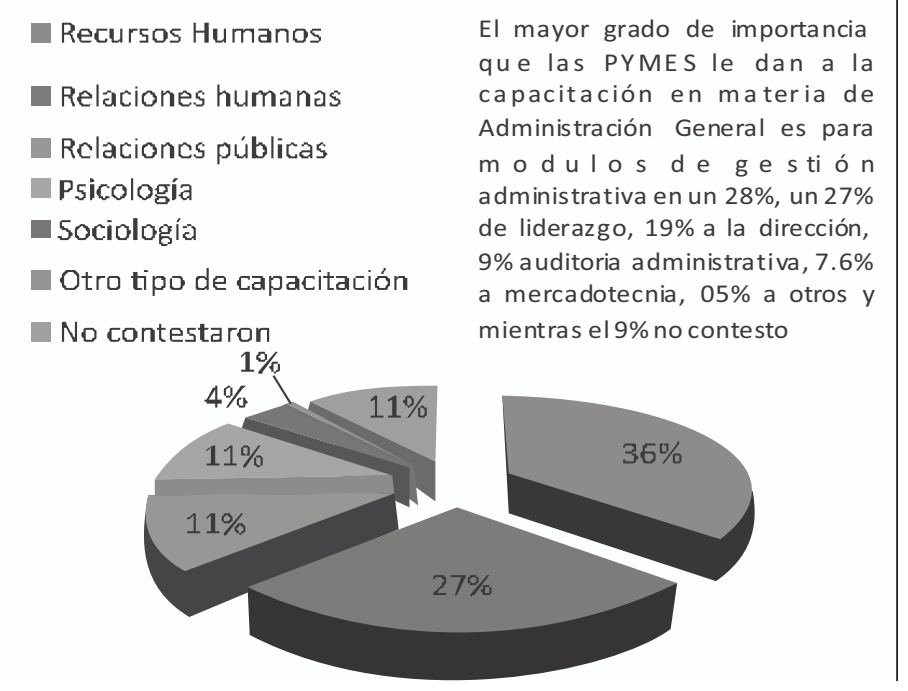

Fuente: Elaboración propia

Figura 13. Importancia que dan las Pymes a las necesidades de capacitación en materia de producción

\section{IMPORTANCIA QUE DAN LAS PYMES A LAS NECESIDADES DE CAPACITACION EN MATERIA DE PRODUCCION}
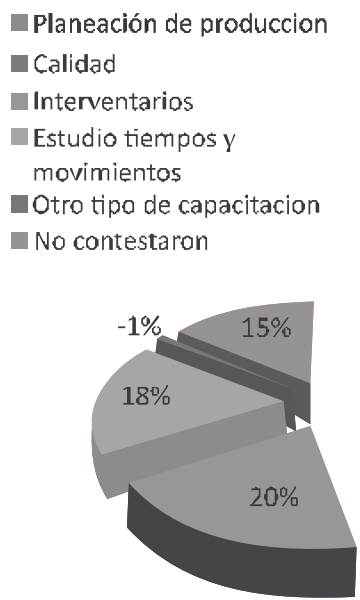

El mayor grado de importancia que las PYMES le dan a la capacitación en materia de producción es para planeación de producción con el $24.6 \%, 22.1 \%$ a calidad, $20.10 \%$ a interventoria, $18.10 \%$ a estudio de tiempos $y$ movimientos, $0.55 \%$ a otro tipo de capacitación y el $14.6 \%$ no contesto.

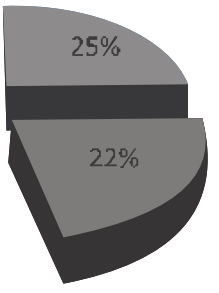

Fuente: Elaboración propia 
LA PUBLICIDAD, UNA ESTRATEGIA DE ÉXITO PARA LAS MICRO, PEQUEÑAS Y MEDIANAS EMPRESAS DE LA REGIÓN CENTRO Y SUROESTE DEL ESTADO DE HIDALGO

Figura 14. Importancia que dan las Pymes a las necesidades de capacitación en materia de informática

\section{IMPORTANCIA QUE DAN LAS PYMES A LAS NECESIDADES DE CAPACITACION EN MATERIA DE INFORMTICA}
Manejo de paqueteria
(microsoft office)
Programación
Aspel
- Otro tipo de capacitacion
no contestaron

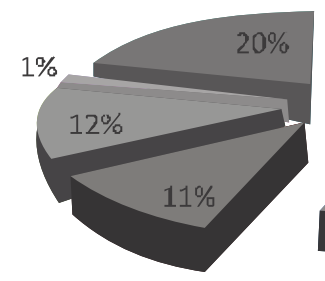

El mayor grado de importancia que las PYMES le dan a la capacitación en materia de informática es para manejo de paquetería con un $56 \%$, programación con un $12 \%$, un $11 \%$, a Aapel, un $1 \%$ a otro tipo de capacitación y el $20 \%$ no contesto.

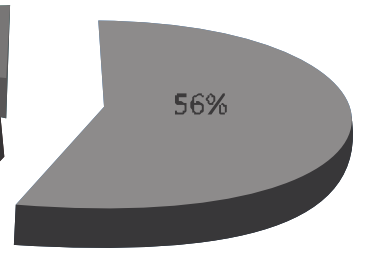

Fuente: Elaboración propia

Figura 15. Importancia que dan las Pymes a las necesidades de capacitación en materia de idiomas

\section{IMPORTANCIA QUE DAN LAS PYMES A LAS NECESIDADES DE CAPACITACION EN MATERIA DE INFORMTICA}

Ingles
Frances
Otro
No contestaron

El mayor grado de importancia que las PYMES le dan a la capacitación en materia de idiomas es para ingles con un $76 \%$, frances con un $0.5 \%$, otro con un $0.5 \%$ y en un $23 \%$ no contestaron

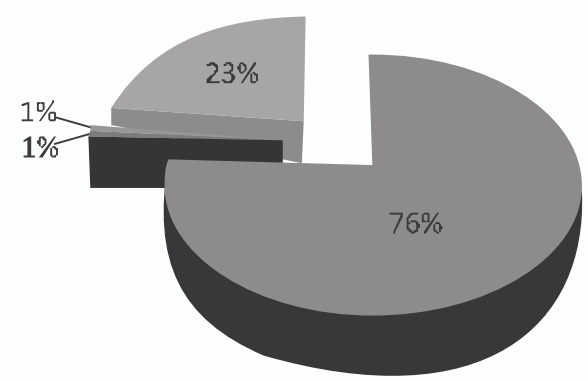

Fuente: Elaboración propia 


\section{Conclusiones de la encuesta}

Una vez obtenidos los resultados podemos concluir que en la región suroeste del estado de Hidalgo en relación con los datos arrojados, podemos observar que la mayoría de las PyMEs:

$>$ Pertenecen al sector comercial.

$>$ Cuentan con un promedio de entre uno y diez trabajadores.

$>$ Requieren de los servicios de capacitación en el área de la administración y mercadotecnia.

$>$ No utilizaron servicios de capacitación en el ámbito de la administración y mercadotecnia. El tipo de capacitación que más se utilizo el año pasado fueron los cursos.

$>$ Capacitan más a los trabajadores en el área de personal.

> Utilizan los exámenes para evaluar el impacto de la capacitación que ofrecen a su empresa.

$>$ Incorporarían un programa de capacitación y desarrollo en materia de administración de empresas y mercadotecnia.

$>$ Necesitan capacitación de modelos de gestión administrativa en materia de Admón. General, para el buen desempeño de su empresa.

$>$ Necesitan capacitación en contabilidad básica.

$>$ En materia legal se le da mayor importancia a la necesidad de capacitación al derecho laboral.

$>$ Necesitan capacitación para recursos humanos, en materia de personal.

$>$ Consideran importante la capacitación en la planeación de la producción.

$>$ Dan prioridad a la capacitación en el manejo de paquetería (Microsoft office).

$>$ El idioma inglés es de gran importancia para las necesidades de la empresa por lo que se necesita de una capacitación.

En relación a que le dan mayor prioridad a capacitación a materias como derecho laboral, contabilidad básica, en materia de personal, en planeación de la producción, al uso de la paquetería de Office, y al idioma inglés, es bastante malo porque son materias básicas que permiten el buen funcionamiento de la empresa, que por lo tanto en el momento en que el personal no se encuentra capacitado o peor aun no cuentan con los conocimientos necesarios de estas áreas. De tal manera que, aunque la dirección y planeación de la empresa sea excelente sin el buen funcionamiento del personal no se puede llegar al cumplimiento de objetivos y peor aún no se llega a tener una expansión de la empresa. 
LA PUBLICIDAD, UNA ESTRATEGIA DE ÉXITO PARA LAS MICRO, PEQUEÑAS Y MEDIANAS EMPRESAS DE LA REGIÓN CENTRO Y SUROESTE DEL ESTADO DE HIDALGO

Si analizamos desde otro enfoque, este tipo de empresas necesitan capacitación urgente y bien dada por que de nada sirve que las empresas soliciten capacitación y que esta no sea bien implantada y que genere que los empleados no aprovechen esto por lo que se necesita implementar la capacitación eficaz y eficientemente.

Así como si los empresarios quieren llegar a expandir sus empresas o el simple hecho de que funcionen como deben de funcionar deben prestar mayor atención al personal y al hecho de que debe estar actualizado y capacitado para que la empresa sea competente en la región y a un mayor nivel.

Para tener un parámetro más amplio podemos observar que gran parte de las PyMEs no contesto en relación a la necesidad de capacitación para algún área de su empresa, lo que nos puede dar un indicio de no piensan en la capacitación del personal, que puede deberse a factores económicos o puede darse el caso de que sea por la falta de conocimiento del beneficio brindado en una capacitación (estas razones son estimaciones pero se pueden apegar a la realidad considerando el entorno externo de estas MiPyMEs).

La frecuencia en que se utilizan los instrumentos implementados para evaluar el impacto de la capacitación que se ofrecen en las MiPyMEs de la región suroeste del estado de Hidalgo fueron los exámenes, los cuales 11 fueron utilizados por el sector comercial y 5 en el sector de servicios. Mientras que por medio del logro de objetivos fueron 3 del sector industrial, 15 del sector comercial y 4 de servicios, con los indicadores de personal uno del sector comercial y uno de otro tipo de sector, a través de indicadores financieros solamente uno del sector comercial y con indicadores de calidad uno del sector industrial y uno del sector comercial, mientras que 3 utilizaron indicadores de mercado en el sector comercial.

Para observar la competitividad de estas empresas en el rubro administrativo y de mercadotecnia hay que analizar la capacitación del año anterior realizada donde de las 201 MiPyMEs 117 no utilizaron servicios de capacitación, mientras que 79 si lo hicieron, con lo que nos damos cuenta del grado de competitividad que es muy bajo por que pocas son las empresas que capacitan a su personal, pero si la mayoría lograra capacitar mayormente a su personal obtendrían mejores resultados y lograrían el cumplimiento de sus objetivos.

Estrategias de comercialización: publicidad; en las micro, pequeñas y medianas empresas de la región centro y suroeste del estado de Hidalgo.

Es necesario aplicar una comunicación publicitaria que apoye a las micro, pequeñas y medianas empresas de la región centro y suroeste del estado de Hidalgo a tener una publicidad efectiva:

- $\quad$ Conocimiento: es necesario que el público consumidor conozca la marca de nuestro producto. Las cosas que no se conocen no se compran; por lo tanto, la empresa debe hacer un esfuerzo para que los consumidores conozcan su marca o producto.

- $\quad$ Comprensión: es toda información que nos permite el conocimiento del producto: de que esta hecho, para que sirve, qué ventajas tiene, cuando dura, su precio, donde vende, etc.

- Convicción: significa presentar el mensaje publicitario dando las ideas o informaciones necesarias que permitan formar una imagen de calidad, de categoría 
y de prestigio hacia el producto para logara en un momento dado la preferencia en el mercado. También los mensajes publicitarios deben ser sugestivos, deben provocar al posible comprador la motivación para hacer deseable el producto.

- $\quad$ Conducta: es la que provocan que venda, es una fuerza vendedora, pero solo impulsa a la acción de venta (no vende), en otra palabras, la publicidad no tiene la responsabilidad de vender.

La publicidad tiene un poder muy grande, pues bien planeada con una investigación de mercados adecuada, con material de punto de venta adecuado, se logra interesar a los consumidores s adquirir nuestro producto.

El poder publicitario incita a los consumidores con escaso potencial económico a fin de que adquieran nuestros productos o servicios, lo cual es bueno, pues se proporcionan a la sociedad un mejor nivel de vida.

Es el manejo adecuado de todos los elementos que participa en la publicidad, como son: los recursos con que cuenta la empresa, investigación de mercados, manejo adecuado de promociones, manejo adecuado de material en el punto de venta, los que coordinados en forma adecuada nos proporcionan el plan de mercadotecnia.

\section{Decisiones de publicidad}

Para emitir daciones con respecto a la publicidad a utilizar es necesario:

a) El cálculo de la cantidad de recursos a emplear en publicidad y el tiempo en que se distribuirá esa cantidad de recursos.

b) Los medios publicitarios a utilizar.

c) El sector del mercado a quien va dirigida la publicidad.

d) También debemos tomar en cuenta las cualidades que contiene el producto a fin de proyectar su imagen al consumidor.

Concluyendo: las decisiones publicitarias están encaminada a:

a) Obtener mayores recursos para la organización

b) A la estrategia de medios. A hacer uso adecuado de los medios publicitarios.

c) A la estrategia promocional

d) A la estrategia creativa; esta actividad se encuentra encaminada a resaltar cualidades del producto al consumidor, como son: color, olor, tamaño, cualidades, etc. 
LA PUBLICIDAD, UNA ESTRATEGIA DE ÉXITO PARA LAS MICRO, PEQUEÑAS Y MEDIANAS EMPRESAS DE LA REGIÓN CENTRO Y SUROESTE DEL ESTADO DE HIDALGO

Grafico 1. Principales decisiones en la publicidad.

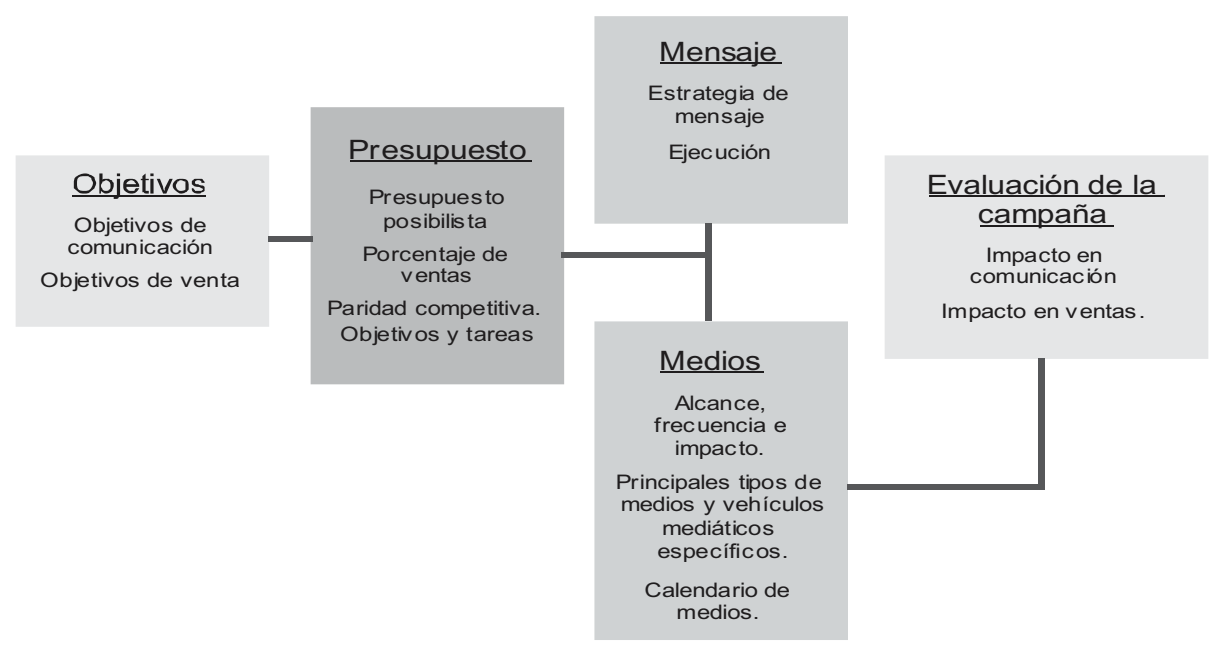

Fuente: Kotler (2006)

\begin{tabular}{|c|c|}
\hline \multicolumn{2}{|c|}{ PUBLICIDAD PARA INFORIMAR } \\
\hline $\begin{array}{l}\text { Informar al mercado sobre un nuevo } \\
\text { producto. } \\
\text { Sugerir nuevos usos para un producto } \\
\text { existente. } \\
\text { Informar al mercado de cambios en los } \\
\text { precios. } \\
\text { Explicar a los consumidores cómo funciona } \\
\text { el producto. }\end{array}$ & $\begin{array}{l}\text { Describir los servicios disponibles. } \\
\text { Rectificar falsas impresiones de los } \\
\text { consumidores. } \\
\text { Reducir los temores de los } \\
\text { consumidores. } \\
\begin{array}{l}\text { Crear imagen de marca de una } \\
\text { empresa. }\end{array}\end{array}$ \\
\hline \multicolumn{2}{|c|}{ PUBLICIDAD PARA CONVENCER (PERSUASIVA) } \\
\hline $\begin{array}{l}\text { Generar preferencia de marca. } \\
\text { Animar a los consumidores a cambiar a una } \\
\text { marca determinada. } \\
\text { Cambiar la percepción que tienen los } \\
\text { consumidores de los atributos del producto. }\end{array}$ & $\begin{array}{l}\text { Convencer a los consumidores de que } \\
\text { compren el producto inmediatamente. } \\
\text { Convencer a los consumidores de que } \\
\text { acepten una oferta de venta. }\end{array}$ \\
\hline PUBLICIDAD PI & RECORDAR \\
\hline $\begin{array}{l}\text { Recordad a los consumidores que podrían } \\
\text { necesitar el producto en un futuro próximo. } \\
\text { Recordar donde comprar el producto. }\end{array}$ & $\begin{array}{l}\text { Mantener el producto en la mente del } \\
\text { consumidor en momentos fuera de } \\
\text { temporada. } \\
\text { Mantener un recuerdo de marca óptimo. }\end{array}$ \\
\hline
\end{tabular}

Fuente: Kotler y otros (2004) 


\section{CONCLUSIONES}

Se concluye que las micro, pequeñas y medianas empresas de la región centro y suroeste del estado de Hidalgo, deberían aprender a utilizar la publicidad como estrategia de comercialización, ya que les permitirá transmitir de manera eficaz y efectiva a través de los distintos medios de comunicación a sus clientes reales y potenciales los atributos y ventajas de sus productos. De esta manera podrán obtener un beneficio en sus ventas y utilidades al implementar nuevas estrategias publicitarias que les permitan posicionarse de manera competitiva en los mercados.

Es importante recordar a las micro, pequeñas y medianas empresas la trascendencia de la publicidad para comercialización de sus productos y servicios, permaneciendo en la mente del consumidor más allá de las campañas de consumo. En el momento de lograr los objetivos de las estrategias de publicidad, deberán contar con cierta ventaja sobre la competencia al mantener un buen nivel de notoriedad, destinadas a recordar y permitir un buen posicionamiento en el mercado (Kotler, 2006).

\section{REFERENCIAS BIBLIOGRÁFICAS}

Ibarra. A. (1999). "El Sistema Normalizado de Competencia Laboral. En Competencia Laboral y Educación Basada en Normas de Competencia”. Editorial Limusa.México. 1999

Instituto nacional de estadística y geografía. (2009). "Resumen de los resultados de los Censos Económicos 2009”. Censos económicos 2009.

Kotler, P., Armstrong, G., Cámara I. D., y Roche C. I. (2004). Marketing. Madrid: Pearson Prentice Hall.

Kotler , P. (2006). “Dirección de Marketing”. Pearson, México.

Martínez, F. (2008). "Análisis estratégico para el desarrollo de la micro, pequeña y mediana empresa". Universidad Autónoma del Estado de Colima.

Nacional Financiera. (2001). "Una Aproximación al Universo "Formal" de MIPYMES para Nacional Financiera”. Dirección de Estudiosos Económicos. México D.F.

Secretaría de Economía. (2012). “Estructura de las PYMES en México”. Recuperado 22/07/2014 de: http://www.economia.gob.mx/conoce-la-se/programas-se

Sistema de Información Empresarial Mexicana. (2010). Estadísticas. Recuperado 25/02/2014 de: http://www.siem.gob.mx/siem/estadisticas 